\title{
Diagnostic accuracy of case-finding questions to identify perinatal depression
}

\author{
Rachel Mann MSc, Joy Adamson PhD, Simon M. Gilbody DPhil
}

Competing interests: None declared.

This article has been peer reviewed.

Correspondence to:

Rachel Mann,

rcm504@york.ac.uk

CMAJ 2012. DOI:10.1503

/cmaj.111213

\begin{abstract}
Background: Guidelines for perinatal mental health care recommend the use of two casefinding questions about depressed feelings and loss of interest in activities, despite the absence of validation studies in this context. We examined the diagnostic accuracy of these questions and of a third question about the need for help asked of women receiving perinatal care.
\end{abstract}

Methods: We evaluated self-reported responses to two case-finding questions against an interviewer-assessed diagnostic standard (DSM-IV criteria for major depressive disorder) among 152 women receiving antenatal care at 26-28 weeks' gestation and postnatal care at 5-13 weeks after delivery. Among women who answered "yes" to either question, we assessed the usefulness of asking a third question about the need for help. We calculated sensitivity, specificity and likelihood ratios for the two case-finding questions and for the added question about the need for help.
Results: Antenatally, the two case-finding questions had a sensitivity of $100 \%$ (95\% confidence interval $[\mathrm{Cl}] 77 \%-100 \%)$, a specificity of $68 \%(95 \% \mathrm{Cl} 58 \%-76 \%)$, a positive likelihood ratio of $3.03(95 \% \mathrm{Cl} 2.28-4.02)$ and a negative likelihood ratio of 0.041 (95\% C 0.003-0.63) in identifying perinatal depression. Postnatal results were similar. Among the women who screened positive antenatally, the additional question about the need for help had a sensitivity of $58 \%(95 \% \mathrm{Cl} 38 \%-76 \%)$, a specificity of $91 \%(95 \% \mathrm{Cl} 78 \%-97 \%)$, a positive likelihood ratio of $6.86(95 \% \mathrm{Cl} 2.16-21.7)$ and a negative likelihood ratio of $0.45(95 \% \mathrm{Cl}$ $0.25-0.80$ ), with lower sensitivity and higher specificity postnatally.

Interpretation: Negative responses to both of the case-finding questions showed acceptable accuracy for ruling out perinatal depression. For positive responses, the use of a third question about the need for help improved specificity and the ability to rule in depression.
$\mathrm{T}$ he occurrence of depressive symptoms during the perinatal period is wellrecognized. The estimated prevalence is $7.4 \%-20 \%$ antenatally ${ }^{1,2}$ and up to $19.2 \%$ in the first three postnatal months. ${ }^{3}$ Antenatal depression is associated with malnutrition, substance and alcohol abuse, poor self-reported health, poor use of antenatal care services and adverse neonatal outcomes. ${ }^{4}$ Postnatal depression has a substantial impact on the mother and her partner, the family, mother-baby interaction and on the longer-term emotional and cognitive development of the baby. ${ }^{5}$

Screening strategies to identify perinatal depression have been advocated, and specific questionnaires for use in the perinatal period, such as the Edinburgh Postnatal Depression Scale, ${ }^{6}$ were developed. However, in their current recommendations, the UK National Screening Committee and the US Committee on Obstetric Practice ${ }^{8}$ state that there is insufficient evidence to support the implementation of universal perinatal screen- ing programs. The initial decision in 2001 by the National Screening Committee to not support universal perinatal screening ${ }^{9}$ attracted particular controversy in the United Kingdom; some service providers subsequently withdrew resources for treatment of postnatal depression, and subsequent pressure by perinatal community practitioners led to modification of the screening guidance in order to clarify the role of screening questionnaires in the assessment of perinatal depression..$^{10}$

In 2007, the National Institute for Health and Clinical Excellence issued clinical guidelines for perinatal mental health care in the UK, which included guidance on the use of questionnaires to identify antenatal and postnatal depression. ${ }^{11}$ In this guidance, a case-finding approach to identify perinatal depression was strongly recommended; it involved the use of two casefinding questions (sometimes referred to as the Whooley questions), and an additional question about the need for help asked of women who 
answered "yes" to either of the initial questions (Box 1).

Useful case-finding questions should be both sensitive and specific so they accurately identify those with and without the condition. The two case-finding questions have been validated in primary care samples ${ }^{12,13}$ and examined in other clinical populations ${ }^{14-16}$ and are endorsed in recommendations by US and Canadian bodies for screening depression in adults. ${ }^{17,18}$ However, at the time the guidance from the National Institute for Health and Clinical Excellence was issued, there were no validation studies conducted in perinatal populations. A recent systematic review ${ }^{19}$ identified one study conducted in the United States that validated the two questions against established diagnostic criteria in 506 women attending wellchild visits postnatally; ${ }^{20}$ sensitivity and specificity of the questions were $100 \%$ and $44 \%$ respectively at four weeks. The review failed to identify studies that validated the two questions and the additional question about the need for help against a gold-standard measure.

We conducted a validation study to assess the diagnostic accuracy of this brief case-finding approach against gold-standard psychiatric diagnostic criteria for depression in a population of women receiving perinatal care.

\section{Methods}

\section{Participants and setting}

We recruited participants during a seven-week period from September 2010 to November 2010 in a maternity unit in a UK National Health Service general hospital, where more than $90 \%$ of the women in the local area receive their antenatal care. We approached women attending the clinic at about 26-28 weeks' gestation for a routine appointment who were also recruited to a large population cohort study (the Born in Bradford study). ${ }^{21}$ Women were excluded from our validation study if they were not participating in the Born in Bradford study, did not speak English, were not literate, were planning to move from the Bradford area and were less than 18 years old at delivery.

\section{Screening measures}

\section{Index test}

The two brief case-finding questions recommended in the UK perinatal clinical guidelines ${ }^{11}$ were included in a self-administered questionnaire in written format. Participants were required to indicate "yes" or "no" in response to each question. A "yes" response to either question was considered a positive screen.
To assess the usefulness of the addition of a third question about the need for help, we considered a "yes" response to either case-finding question plus a "yes" response to the third question to be a positive screen.

\section{Diagnostic gold standard}

To confirm the presence or absence of a current depressive episode, the DSM-IV (Diagnostic and Statistical Manual of Mental Disorders, fourth edition) diagnostic criteria for major depressive disorder were administered in an interview by telephone. ${ }^{22}$ Guidance for the administration and interpretation of the criteria was taken from the Structured Clinical Interview for DSM-IV-Clinical Version. ${ }^{23}$ Semistructured questions to identify depressive symptoms were asked in a format suitable for verbal interview ${ }^{24}$ and have previously been shown to be valid when used over the telephone or face to face. ${ }^{25,26}$ The interview questions and DSM-IV criteria used in our study are available in Appendix 1 (www.cmaj.ca/lookup/suppl /doi:10.1503/cmaj.111213/-/DC1).

Participants who did not meet the criteria for major depressive disorder but who had either depressed mood or anhedonia and met one other criterion for major depressive disorder were considered to have minor depression.

\section{Procedure}

The study was conducted in two phases in order to validate the utility of the questions in the antenatal and postnatal periods, as recommended by National Institute for Health and Clinical Excellence. ${ }^{11}$ The study is reported according to the STARD (Standards for the Reporting of Diagnostic accuracy studies) statement. ${ }^{27}$

During the antenatal phase, participants were recruited sequentially while attending a routine antenatal appointment at about 26-28 weeks' gestation (mean 26.7 weeks, standard deviation 1.7). Participants completed the self-administered questionnaire in private; a researcher (R.M.) was available to answer questions if necessary.

During the postnatal phase, a copy of the questionnaire with a covering letter was mailed

Box 1: Case-finding questions recommended for the identification of perinatal depression ${ }^{10}$

- "During the past month, have you often been bothered by feeling down, depressed or hopeless?"

- "During the past month, have you often been bothered by having little interest or pleasure in doing things?"

- A third question should be considered if the woman answers "yes" to either of the initial screening questions: "Is this something you feel you need or want help with?" 
to the participants at about five to six weeks postnatally. The women were asked to return the completed questionnaire within seven days as well as a reply form to indicate a time and date when they would be available for the diagnostic interview by telephone. If necessary, nonrespondents were mailed another copy of the questionnaire and covering letter, sent up to two reminder letters and contacted once by telephone.

Within 14 days after completion of each of the case-finding questionnaires, a diagnostic interview was conducted by telephone by one of us (R.M.). The researcher had previous clinical and research experience with the administration

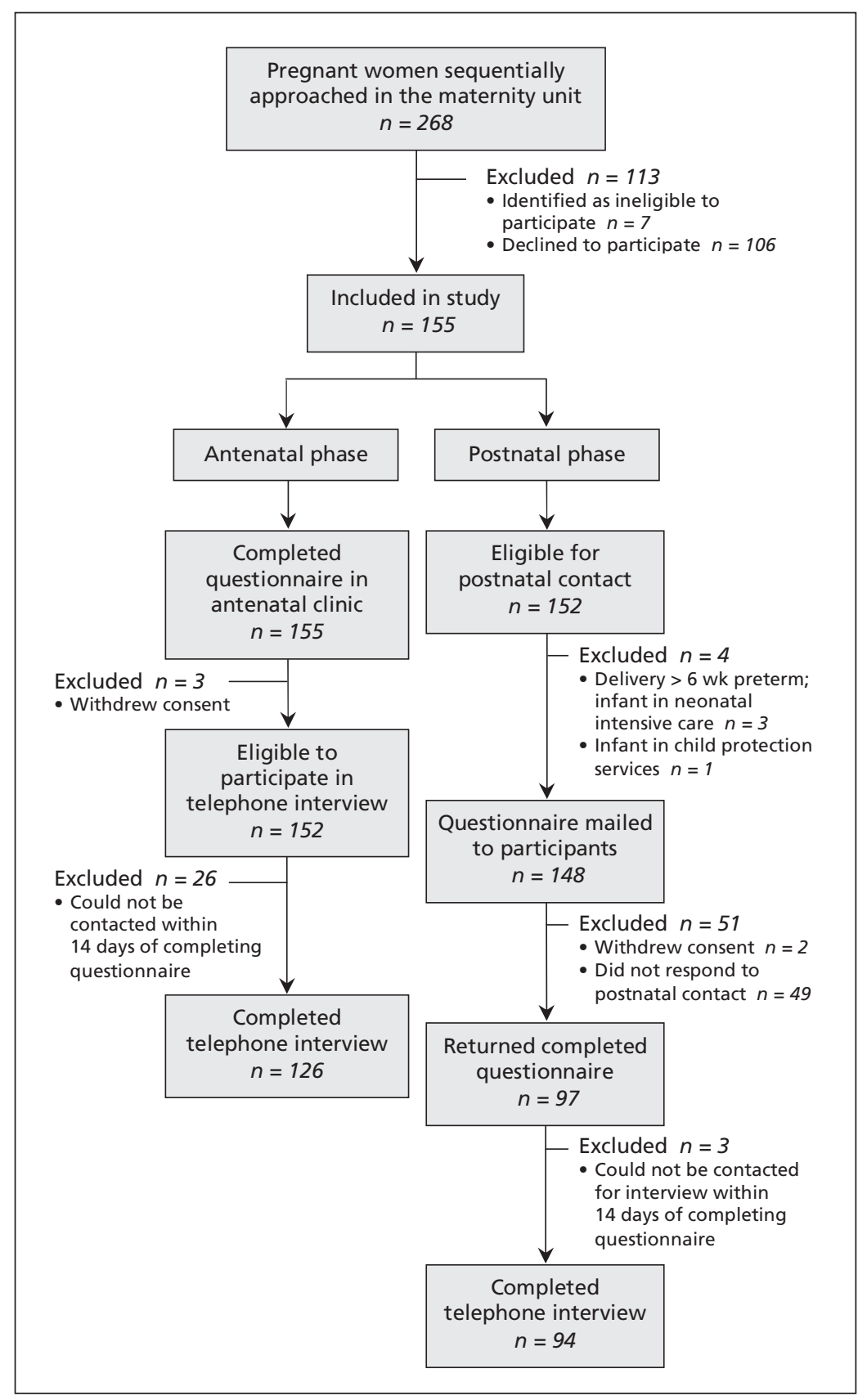

Figure 1: Flow of patients through the study. of diagnostic interviews. To prevent review bias, she was unaware of the participant's responses to the case-finding questionnaire before the interview.

\section{Statistical analysis}

The calculation of the sample size was based on the method developed by Flahault and colleagues. ${ }^{28}$ For an expected sensitivity of $95 \%$ with 0.95 probability that the minimum acceptable lower $95 \%$ confidence interval (CI) limit would not fall below $70 \%$, and where the prevalence of antenatal and postnatal depression was estimated to be up to $20 \%$, we determined that a sample size of 120 was required.

We calculated the sensitivity, specificity, and positive and negative likelihood ratios, and the associated $95 \%$ CIs, using the online calculator available on the University of Toronto's website for the Centre for Evidence-Based Medicine. ${ }^{29}$ Sensitivity, specificity and likelihood ratios were interpreted according to established recommendations. ${ }^{30}$ If a $2 \times 2$ cell contained zero, the value of 0.5 was added in order to calculate likelihood ratios and associated $95 \%$ CIs. ${ }^{31}$ Statistical significance was interpreted at the 5\% level. Additional analyses were conducted with the use of PASW Statistics 18 (formerly SPSS Statistics).

\section{Results}

Figure 1 displays the selection and flow of participants through the study. The 152 eligible participants and the 106 women who were eligible but who declined to participate did not differ significantly in terms of age $(p=0.2)$ or ethnic background $(p>0.9)$.

\section{Antenatal phase}

A total of 152 women completed the casefinding questionnaire during their antenatal visit. The sample was diverse, with a varied range of ethnic backgrounds and levels of education (Table 1). Of these women, 126 (82.9\%) completed the telephone interview to confirm the presence or absence of depression. ${ }^{27}$ These participants and the 26 women who could not be contacted for the interview did not differ significantly in terms of age $(p=0.1)$ or ethnic background $(p=0.46)$.

The proportion of women who met the criteria for depression (minor and major) during the antenatal phase was $13.5 \%$ (95\% CI $8.3 \%$ $21 \%)$. The sensitivity, specificity and likelihood ratios of the two case-finding questions and the additional question about the need for help are reported in Table 2. A positive response to either of the case-finding questions (positive screen) showed acceptable sensitivity (100\%); all of the 
women with depression were classified correctly, with no false-negative results. The negative likelihood ratio of 0.041 indicated acceptable accuracy to rule out the presence of depression given a negative response to both questions.

Among the women with a positive screen, use of the additional question about the need for help improved specificity to $91 \%$. The positive likelihood ratio of 6.86 showed that the additional question was moderately good at ruling in antenatal depression.

\section{Postnatal phase}

Of the 152 women eligible for postnatal followup contact, 4 were excluded because of a breach of study protocol (Figure 1). Of the remaining 148 women to whom the questionnaire was mailed, 2 withdrew consent and 97 returned a completed questionnaire within 13 weeks of delivery (response rate 66\%). A total of 94 (97\%) completed the telephone interview.

The mean number of weeks from delivery to participation in the postnatal diagnostic interview was seven weeks and four days (standard deviation [SD] 1.7 weeks). Compared with the 94 women who participated in the postnatal phase of the study, the 52 women who did not respond to postnatal contact were younger, less educated and less likely to be employed (Table 3).

The proportion of participants who met the criteria for depression (minor and major) during the postnatal phase was $19.2 \%$ (95\% CI $12 \%$ $28.9 \%)$. The sensitivity, specificity and likelihood ratios of the two case-finding questions and the additional question about the need for help are reported in Table 2 . The sensitivity of a positive response to either case-finding question was $100 \%$; all of the women with postnatal depression were classified correctly. The negative likelihood ratio of 0.042 showed acceptable accuracy to rule out the presence of postnatal depression given a negative response to both questions.

Among the participants with a positive screen, use of the additional question about the need for help improved specificity to $100 \%$. The positive likelihood ratio of 21.4 showed that a positive response to the additional question was acceptable at ruling in postnatal depression.

\section{Interpretation}

We found that the two case-finding questions endorsed by the National Institute for Health and Clinical Excellence offered a brief, simple and precise approach for identifying perinatal depression. Negative responses to both questions showed acceptable accuracy for ruling out perinatal depression. For positive responses, the use
Table 1: Characteristics of study participants

\begin{tabular}{|c|c|}
\hline Characteristic & $\begin{array}{c}\text { No. }(\%) \text { of patients* } \\
n=152\end{array}$ \\
\hline Age, yr, mean (SD) & $27.4(5.8)$ \\
\hline \multicolumn{2}{|l|}{ Ethnic background } \\
\hline White British & $81(53.3)$ \\
\hline White other & 5 (3.3) \\
\hline Mixed (white and black) & $4(2.6)$ \\
\hline Mixed (white and South Asian) & $3(2.0)$ \\
\hline Black & 6 (3.9) \\
\hline Indian & $5 \quad(3.3)$ \\
\hline Pakistani & $38(25.0)$ \\
\hline Bangladeshi & $5(3.3)$ \\
\hline Other & $5(3.3)$ \\
\hline Primiparous & $73(48.0)$ \\
\hline \multicolumn{2}{|l|}{ Marital status } \\
\hline Married, first marriage & $79(52.0)$ \\
\hline Remarried & $10(6.6)$ \\
\hline Single, never married & $60(39.9)$ \\
\hline Divorced & $3(2.0)$ \\
\hline \multicolumn{2}{|l|}{ Cohabitation } \\
\hline Lives with baby's father & $122(80.3)$ \\
\hline Lives with another partner & $1(0.7)$ \\
\hline In a relationship, not living with partner & $20(13.2)$ \\
\hline Not in a relationship & $9(5.9)$ \\
\hline \multicolumn{2}{|l|}{ Housing } \\
\hline Owns house, paying mortgage/loan & $68(44.7)$ \\
\hline Owns house outright & $13(8.6)$ \\
\hline Rents & $54(35.5)$ \\
\hline Lives rent free & $17(11.2)$ \\
\hline \multicolumn{2}{|l|}{ Highest level of education } \\
\hline None & $22(14.5)$ \\
\hline High school & $35(23.0)$ \\
\hline College or university & $31(20.4)$ \\
\hline Postgraduate school & $49(32.2)$ \\
\hline Other & $12(7.9)$ \\
\hline No response & $3(2.0)$ \\
\hline \multicolumn{2}{|l|}{ Employment status } \\
\hline Currently employed & $94(61.8)$ \\
\hline Previously employed & $34(22.4)$ \\
\hline Never employed & $24(15.8)$ \\
\hline \multicolumn{2}{|l|}{ Smoking status } \\
\hline Current smoker & $22(14.5)$ \\
\hline Former smoker & $42(27.6)$ \\
\hline Never smoked & $88(57.9)$ \\
\hline \multicolumn{2}{|l|}{ Self-reported history of diagnosed depression } \\
\hline No prior diagnosis by general practitioner & $128(84.2)$ \\
\hline$\geq 1$ diagnosed episode of depression & $24(15.8)$ \\
\hline
\end{tabular}


of an additional question about the need for help improved specificity and the ability to rule in perinatal depression.

This approach has important implications for clinical practice. Use of the case-finding questions should be considered in the context of a triage test, rather than as a replacement test to existing methods of assessment. ${ }^{32}$ Triage tests are simple and noninvasive, have no wait time and do not aim to improve the diagnostic pathway; instead, they reduce the number of patients who need further assessment. The benefit of using the brief case-finding approach in clinical settings where routine perinatal care takes place is not necessarily to diagnose perinatal depression per se. It would, however, reduce the number of women who need extensive clinical assessment or evaluation with much longer questionnaires, such as the Edinburgh Postnatal Depression Scale ${ }^{6}$ or the Patient Health Questionnaire-9,,$^{33}$ by more than $50 \%$. The use of good psychometric instruments with acceptable discriminative properties is a necessary but not sufficient step for use in routine practice in larger population-based screening strategies, and the adoption of screening for disorders such as postnatal depression should be based on evidence of benefit derived from randomized trials. In other areas, this has not been shown to be an effective strategy, and screening should therefore not be considered without a wider consideration of the policy implications, costs and benefits. ${ }^{34}$

The sensitivity of the two case-finding questions in our antenatal and postnatal validation study $(100 \%)$ was the same as that in a previous postnatal validation study, ${ }^{20}$ providing further evidence of a simple approach to rule out perinatal depression. The number of falsepositive responses to the two questions was substantial in the previous postnatal study and in our study.

Our results should be considered alongside the results of a similar validation study in a primary care population. ${ }^{13}$ The increased specificity of an additional question about the need for help effectively discriminated between positive screens. In our study, the added question resulted in the number of false-positive responses in the postnatal phase dropping to zero.

Increased specificity compromises sensitivity, however, and increases the risk of depression being missed (false negative). Arroll and colleagues $^{13}$ reported increased specificity and unchanged sensitivity (96\%) when respondents answered either "yes" or "yes, but not today" or "no" to the additional question about the need for help. They identified 12 false-negative cases but did not refer to this issue in their discussion. In our study, the answer to the additional question was dichotomized as "yes" or "no"; sensitivity was reduced, and the number of falsenegative cases was 7 in the antenatal phase and 11 in the postnatal phase. Poor discrimination between true-negative and false-negative cases in practice may mean that patients with depression are effectively lost to follow-up. The benefit of the additional question about the need for help was therefore not conclusive in our perinatal sample.

Table 2: Performance of two case-finding questions (index test) and an additional question about the need for help in identifying perinatal depression

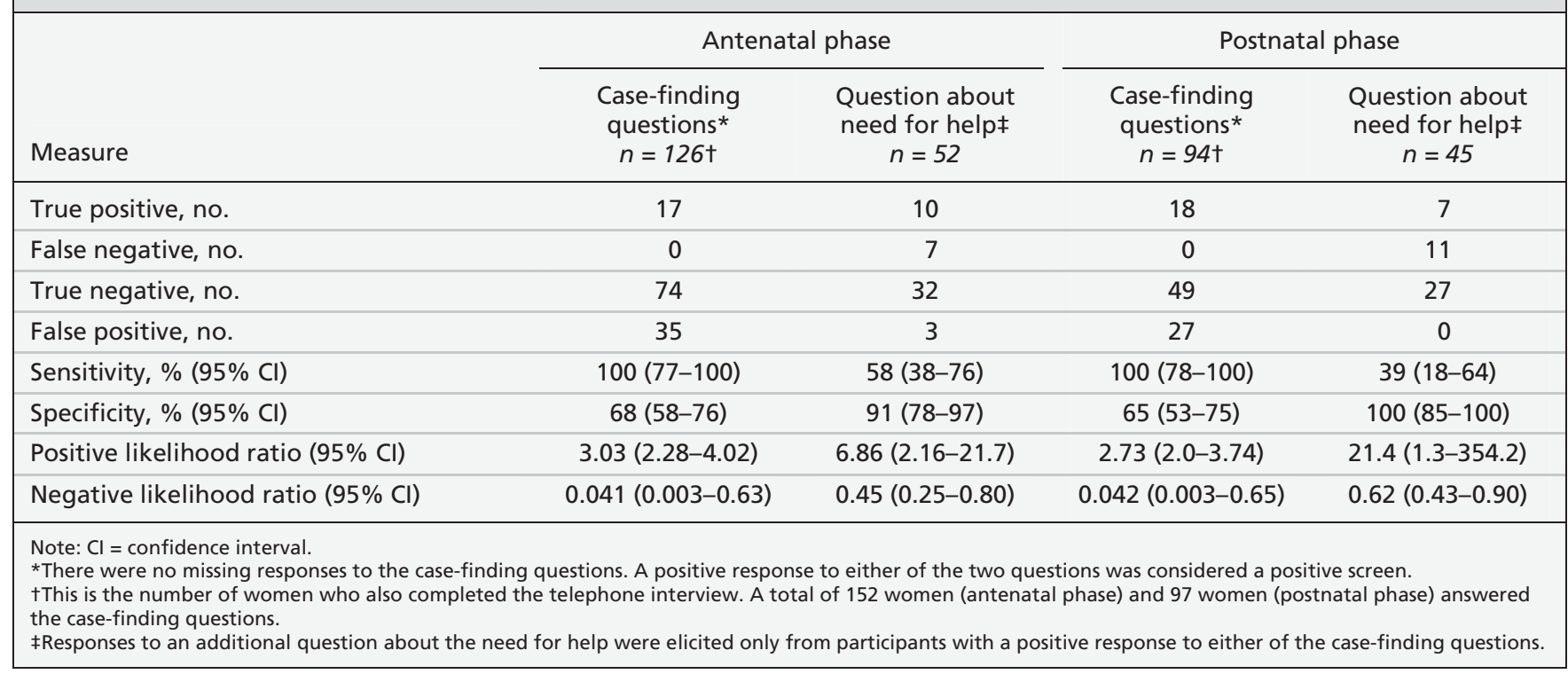


Table 3: Characteristics of the 94 women who participated in the postnatal phase of the study and the 52 women who did not

\begin{tabular}{|c|c|c|c|}
\hline \multirow[b]{2}{*}{ Characteristic } & \multicolumn{2}{|c|}{$\begin{array}{l}\text { Participation in postnatal phase; } \\
\text { no. }(\%) \text { of women* }\end{array}$} & \multirow[b]{2}{*}{$p$ value§ } \\
\hline & $\begin{array}{l}\text { Participatedt } \\
\quad n=94\end{array}$ & $\begin{array}{l}\text { Did not participate } \neq \\
\qquad n=52\end{array}$ & \\
\hline Age, yr, mean (SD) & $28.5(6.2)$ & $25.4(4.4)$ & 0.002 \\
\hline White & $58(62)$ & $25(48)$ & 0.1 \\
\hline Primiparous & $48(51)$ & $21(40)$ & 0.2 \\
\hline Married & $60(64)$ & $25(48)$ & 0.06 \\
\hline Living with baby's father or another partner & $81(86)$ & $38(73)$ & 0.05 \\
\hline House owner & $47(50)$ & $18(35)$ & 0.07 \\
\hline Attended school (any level) & $76(81)$ & $33(64)$ & 0.02 \\
\hline Currently employed & $65(69)$ & $25(48)$ & 0.01 \\
\hline Nonsmoker during pregnancyף & $84(89)$ & $43(83)$ & 0.3 \\
\hline $\begin{array}{l}\text { Self-reported history of } \geq 1 \text { diagnosed } \\
\text { episodes of depression }\end{array}$ & $16(17)$ & $6(12)$ & 0.4 \\
\hline \multicolumn{4}{|c|}{$\begin{array}{l}\text { Note: SD = standard deviation. } \\
\text { *Unless stated otherwise. } \\
\text { †Completed the questionnaire in both the antenatal and postnatal phases and completed the telephone interview. } \\
\text { †Completed the questionnaire in the antenatal phase only ( } 49 \text { did not respond to any postnatal contact and } 3 \text { did not complete } \\
\text { the postnatal telephone interview). } \\
\S \text { Values were compared by means of the } t \text { test and } \chi^{2} \text { test. } \\
\text { ๆIncludes former smokers and those who never smoked. }\end{array}$} \\
\hline
\end{tabular}

\section{Limitations}

Twenty-six women in the antenatal phase did not complete the diagnostic telephone interview. However, these women did not differ significantly from the 126 who were interviewed in terms of age and ethnic background.

In the postnatal phase, the 52 women who did not respond to postnatal contact were significantly younger, less educated and less likely to be employed than those who returned the questionnaire. This is an important limitation in our sample. In terms of age, it may reflect difficulties surrounding the competing demands associated with the transition to motherhood for younger mothers. In addition, some women may not have responded because of postnatal depressive symptoms.

\section{Implications for future research}

Further studies are warranted because our study was limited to the third trimester and first three postnatal months. Studies that involve other perinatal populations, include other trimesters and have longer postnatal follow-up are required. Specific strategies to retain nonrespondents, especially those who are younger and less educated, might be considered. Validation of the case-finding approach among pregnant women who do not speak English and those who are less than 18 years old is needed. Finally, the effect of the questions on outcomes of perinatal care warrants evaluation.

\section{Conclusion}

The brevity of the case-finding questions has substantial appeal for the identification of perinatal depression in frontline health care services. In our study, the use of specific case-finding questions had acceptable validity in the perinatal setting. The ability to rule out depression would help to substantially reduce the number of women needing more extensive evaluation of their antenatal and postnatal mental health issues. Identification of perinatal depression is important but represents only the first step. It must be followed by confirmation of the diagnosis and appropriate treatment or referral. Ultimately, the findings of our study may assist the utility of clinical guidelines that advocate the brief casefinding approach for the identification of perinatal depression.

\section{References}

1. Bennett HA, Einarson A, Taddio A, et al. Prevalence of depression during pregnancy: systematic review [published erratum in Obstet Gynecol 2004;103:1344]. Obstet Gynecol 2004;698:709.

2. Marcus SM, Flynn H, Blow F, et al. Depressive symptoms among pregnant women screened in obstetrics settings. $J$ Womens Health (Larchmt) 2003;12:373-80.

3. Gavin NI, Gaynes BN, Lohr KN, et al. Perinatal depression: a systematic review of prevalence and incidence. Obstet Gynecol 2005; 106:1071-83.

4. Marcus SM. Depression during pregnancy: rates, risks and consequences. Can J Clin Pharmacol 2009;16:e15-22.

5. Murray L, Cooper PJ. The impact of postpartum depression on child development. Int Rev Psychiatry 1996;8:55-63.

6. Cox JL, Holden J, Sagovsky R. Detection of postnatal depres- 
sion: development of the 10-item Edinburgh Postnatal Depression Scale. Br J Psychiatry 1987;150:782-6.

7. Hill C. An evaluation of screening for postnatal depression agains NSC criteria. London (UK): UK National Screening Committee 2010. Available: www.screening.nhs.uk/postnataldepression (2011 Nov. 18).

8. American College of Obstetricians and Gynecologists. Committee on Obstetric Practice. Committee opinion no. 453: Screening for depression during and after pregnancy. Obstet Gynecol 2010; 115(2 pt 1):394-5.

9. Shakespeare J. Evaluation of screening for postnatal depression against NSC handbook criteria. London (UK): National Screening Committee; 2001.

10. Shakespeare J. Screening: the role and recommendations of the UK NSC. In: Henshaw C, Elliott S, editors. Screening for perinatal depression. London (UK) and Philadephia (PA): Jessica Kingsley Publishers; 2005: p. 32-33.

11. National Institute for Health and Clinical Excellence. Antenatal and postnatal mental health: clinical management and service guidance. London (UK): The Institute; 2007.

12. Whooley MA, Avins AL, Miranda J, et al. Case-finding instruments for depression: two questions are as good as many. J Gen Intern Med 1997;12:439-45.

13. Arroll B, Goodyear-Smith F, Kerse N, et al. Effect of the addition of a "help" question to two screening questions on specificity for diagnosis of depression in general practice: diagnostic validity study. BMJ 2005;331:884.

14. Mohr DC, Hart SL, Julian L, et al. Screening for depression among patients with multiple sclerosis: two questions may be enough. Mult Scler 2007;13:215-9.

15. McManus D, Pipkin SS, Whooley MA. Screening for depression in patients with coronary heart disease (data from the Heart and Soul Study). Am J Cardiol 2005;96:1076-81.

16. Mallen CD, Peat G. Screening older people with musculoskeletal pain for depressive symptoms in primary care. $\mathrm{Br} \mathrm{J} \mathrm{Gen}$ Pract 2008;58:688-93.

17. US Preventive Services Task Force (USPSTF). Screening for depression: recommendations and rationale. Ann Intern Med 2002; 136:760-4.

18. MacMillan HL, Patterson CJS, Wathen CN; The Canadian Task Force on Preventive Health Care. Screening for depression in primary care: recommendation statement from the Canadian Task Force on Preventive Health Care. CMAJ 2005;172:33-5.

19. Mann R, Gilbody S. Validity of two case finding questions to detect postnatal depression: A review of diagnostic test accuracy. J Affect Disord 2011;133:388-97.

20. Gjerdingen D, Crow S, McGovern P, et al. Postpartum depression screening at well-child visits: validity of a 2-question screen and the PHQ-9. Ann Fam Med 2009;7:63-70.

21. Raynor P; Born in Bradford Collaborative Group. Born in Bradford, a cohort study of babies born in Bradford, and their parents: protocol for the recruitment phase. BMC Public Health 2008; 8:327.

22. American Psychiatric Association. Diagnostic and statistical manual of mental disorders. 4th ed. Washington (DC): The Association; 1994. p. 356, 777.

23. First MB, Spitzer RL, Gibbon M, et al. Structured clinical interview for DSM-IV - clinical version (SCID-CV) (user's guide and interview). Washington (DC): American Psychiatric Press; 1997.
24. Williams JW, Noel PH, Cordes JA, et al. Is this patient clinically depressed? JAMA 2002;287:1160-70.

25. Rohde P, Lewinsohn P, Seeley J. Comparability of telephone and face-to-face interviews in assessing axis I and II disorders. Am J Psychiatry 1997;154:1593-8.

26. Simon GE, Revicki D, VonKorff M. Telephone assessment of depression severity. J Psychiatr Res 1993;27:247-52.

27. Bossuyt PM, Reitsma JB, Bruns DE, et al. Towards complete and accurate reporting of studies of diagnostic accuracy: the STARD initiative. BMJ 2003;326:41-4.

28. Flahault A, Cadilhac M, Thomas G. Sample size calculation should be performed for design accuracy in diagnostic test studies. J Clin Epidemiol 2005;58:859-62.

29. Centre for Evidence-Based Medicine. Stats calculator. Toronto (ON): University of Toronto. Available: http://ktclearinghouse .ca/cebm/toolbox/statscalc (2011 Nov. 18).

30. Doust J. Diagnosis in general practice: using probabilistic reasoning. BMJ 2009;339(b3823).

31. Glas AS, Lijmer JG, Prins MH, et al. The diagnostic odds ratio: a single indicator of test performance. J Clin Epidemiol 2003; 56:1129-35.

32. Bossuyt PM, Reitsma JB, Bruns DE, et al. Towards complete and accurate reporting of studies of diagnostic accuracy: the STARD initiative. BMJ 2003;326:41-4.

33. Spitzer RL, Kroenke K, Williams JBW. Validation and utility of a self-report version of PRIME-MD: the PHQ primary care study. Primary Care Evaluation of Mental Disorders. Patient Health Questionnaire. JAMA 1999;282:1737-44.

34. Gilbody S, Sheldon T, Wessely S. Should we screen for depression? BMJ 2006;332:1027-30.

Affiliations: From the Department of Health Sciences (Mann, Adamson, Gilbody), and the Hull York Medical School (Mann, Gilbody), University of York, York, United Kingdom

Contributions: Rachel Mann designed the study, undertook the data collection and interviews, analyzed the data and drafted the final manuscript. Simon M. Gilbody and Joy Adamson advised in the design and conduct of the study, and commented on and helped draft the final manuscript. All of the authors approved the final version submitted for publication. Rachel Mann is the guarantor.

Funding: Primary research costs were funded by a research fellowship from Hull York Medical School. The funder had no role in the study design; the collection, analysis and interpretation of the data; the writing of the report; or the decision to submit the article for publication.

Acknowledgements: The authors thank the women who participated in this study. They also thank Dr. Catherine Hewitt for statistical advice. They are grateful to the families who took part in the Born in Bradford study, to the midwives for their help in recruiting the participants, to the pediatricians and health visitors, and to the Born in Bradford team, which included interviewers, data managers, laboratory staff, clerical workers, research scientists, volunteers and managers. 